\title{
«Tiefe des Ansprechens» nach standardmäßiger systemischer Chemotherapie bei metastasiertem kolorektalem Karzinom ist mit Gesamtüberleben assoziiert
}

\author{
Schlüsselwörter \\ Metastasiertes kolorektales Karzinom - Tiefe des Ansprechens. \\ Progressionsfreies Überleben · Gesamtüberleben
}

\begin{abstract}
Zusammenfassung
Hintergrund: Die Feststellung des Ansprechens ist ein wichtiger Aspekt in der chemotherapeutischen Behandlung von Patienten mit metastasiertem kolorektalem Karzinom (metastatic colorectal cancer; $m C R C$ ). Die «Tiefe des Ansprechens» (deepness of response; DpR), definiert als maximal erreichte Rate der Reduktion der Tumorlast im Vergleich zum Behandlungsbeginn, wurde kürzlich als neuer hypothetischer Parameter im Zusammenhang mit dem Gesamtüberleben (overall survival; OS) unter Erstlinien-Chemotherapie plus Cetuximab bei mCRC vorgeschlagen. Wir untersuchten, ob dieses Konzept auch auf andere Standard-ChemotherapieSchemata bei mCRC übertragbar ist.
\end{abstract}

Methoden: Wir analysierten die Daten von mCRC-Patienten, die zwischen Juni 2005 und März 2015 in unserer Klinik mit einer systemischen Erstlinien-Chemotherapie mit FOLFOX, CapeOX oder FOLFIRI (plus Biologika) behandelt worden waren. Retrospektiv erhoben wir Daten wie klinisch-pathologische Parameter, von Metastasen befallene Organe, das angewandte ChemotherapieSchema, das beste Ansprechen laut RECIST v1.1, das progressionsfreie Überleben (progression-free survival; PFS) sowie das OS von Patienten, bei denen eine Tumorverkleinerung eingetreten war.
Die DpR wurde berechnet als unidimensionale maximale Reduktionsrate der messbaren Tumoren. Wir untersuchten die Assoziation zwischen der DpR und dem Überleben.

Ergebnisse: Von den 156 Patienten, die eine Erstlinien-Chemotherapie erhielten, wurde bei 63 eine Tumorverkleinerung festgestellt (41 hiervon waren Männer; Altersmedian 62 Jahre). Eine Vollremission wurde bei 6 Patienten, eine Teilremission bei 42 und eine Stabilisierung der Erkrankung bei 15 erreicht. Die mediane DpR betrug 44,2\% und wurde analog zu vorhergehenden Berichten als Cut-off verwendet. Eine DpR $\geq 45 \%$ (31 Patienten) korrelierte mit einem längeren PFS (Median 16,4 vs. 8,1 Monate bei DpR < 45\%; $p=0,006$ ) und OS (Median 58,6 vs. 30,9 Monate bei DpR < 45\%; $p=0,041$ ). Im Hinblick auf die nachfolgende Chemotherapie bestand kein wesentlicher Unterschied zwischen den Patienten mit $\mathrm{DpR} \geq 45 \%$ und denen mit $\mathrm{DpR}<45 \%$.

Schlussfolgerung: Bei verschiedenen initialen systemischen Chemotherapie-Schemata zur Erstlinienbehandlung des mCRC korrelierte die DpR mit dem OS.

(c) 2016 S. Karger GmbH, Freiburg

\section{Originalartikel}

'Deepness of Response' Is Associated with Overall Survival in Standard Systemic Chemotherapy for Metastatic Colorectal Cancer

Hiroaki Nozawa Takeshi Nishikawa Toshiaki Tanaka Junichiro Tanaka Tomomichi Kiyomatsu Kazushige Kawai Keisuke Hata Shinsuke Kazama Hironori Yamaguchi Soichiro Ishihara Eiji Sunami Joji Kitayama Toshiaki Watanabe

\section{KARGER}

Fax +497614520714

information@karger.com

www.karger.com 
PD Dr. Sylvie Lorenzen

sylvie.lorenzen@mri.tum.de

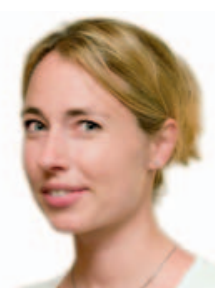

\section{Transfer in die Praxis}

Das vorrangige Ziel einer Erstlinientherapie beim metastasierten Kolorektalkarzinom (metastatic colorectal cancer; mCRC) sollte die Heilung oder die Verlängerung des Überlebens sein. Eine effiziente frühe Tumorschrumpfung (early tumor shrinkage; ETS) sowie die Tiefe des Ansprechens (depth of response; DpR), definiert als maximale Tumorschrumpfung gegenüber dem Behandlungsbeginn, sind dabei wesentliche Faktoren für den Therapieerfolg, weil dadurch die Möglichkeiten für eine Resektion der Metastasen verbessert und auch die vom Tumor verursachten Symptome vermindert werden können. Diese über die Response Evaluation Criteria in Solid Tumors (RECIST) hinausgehenden - tumordynamischen Parameter scheinen den Gesamtnutzen und insbesondere die Sensitivität einer Therapie besser abzubilden als die bislang gebräuchliche, alleinige radiologische Beurteilung des Ansprechens. Die prognostische Wertigkeit von ETS als guter prognostischer Faktor für Verläufe von Patienten mit mCRC wurde kürzlich in einer Meta-Analyse bestätigt [1].

Eine Reihe von klinischen Studien konnte einen Überlebensvorteil für Patienten mit RAS-Wildtyp-mCRC zeigen, die einen gegen den epidermalen Wachstumsfaktor (epidermal growth factor receptor; EGFR) gerichteten Antikörper in Kombination mit einer Chemotherapie als Erstlinientherapie erhalten hatten. Die Auswertungen der FIRE-3- [2, 3] und der Peak-Studie [4] ergaben, dass sowohl ein ETS als auch die DpR mit einem verbesserten progressionsfreien Überleben (PFS) und Gesamtüberleben (OS) assoziiert sind. Beide Studien zeigten übereinstimmend, dass die Kombination einer Chemotherapie mit einem Anti-EGFR-Antikörper den Anteil von Patienten mit ETS erhöht und die Geschwindigkeit der Tumorantwort verbessert im Vergleich zu einer Chemotherapie in Kombination mit einer gegen den VEGF (= vascular endothelial growth factor) gerichteten Therapie.

In der hier vorgestellten monozentrischen Analyse aus Japan wurde die DpR als prognostischer Parameter bei Patienten mit mCRC, die eine Oxaliplatin- oder Irinotecan-basierte Erstlinientherapie mit oder ohne Antikörper im Zeitraum von 2005 bis 2015 erhalten hatten, retrospektiv untersucht. Von 156 analysierten Patienten erreichten 63 Patienten ein Ansprechen, definiert als Tumorvolumen-Reduktion $<30 \%$. Bei den anderen
93 Patienten zeigten die Tumore entweder einen Progress, ein Größenwachstum von $<20 \%$ oder es waren keine Angaben zum Ansprechen verfügbar. Nach diesen Kriterien definierte «Nonresponder» zeigten signifikant häufiger eine Wachstumslokalisation im rechtsseitigen Kolon sowie ein T4-Stadium.

Insgesamt bestätigt diese Arbeit vorangegangene Untersuchungen $[2,5]$ darin, dass die $D p R$, in dieser Analyse definiert mit einem Schwellenwert von $\geq 45 \%$, prädiktiv für ein verbessertes PFS und OS ist. Dabei scheint laut den Autoren die DpR unabhängig von der verwendeten Chemotherapie/Antikörper-Kombination zu sein. Allerdings erlaubt diese Analyse aufgrund der geringen Fallzahlen, insbesondere der mit Anti-EGFR-Antikörpern behandelten Patienten $(n=23)$, keinen Vergleich bezüglich einer unterschiedlichen Effizienz der verwendeten Chemotherapie-Protokolle. Der Einfluss auf die Prognose durch Art und Anzahl der Folgetherapien (fast alle Patienten (96\%) erhielten eine Zweitlinientherapie) wurde ebenso aus der Untersuchung ausgeschlossen.

Die Autoren schließen aus ihrer Analyse, dass die DpR unter einer Erstlinientherapie als prädiktiver Surrogatmarker für das PFS und OS geeignet ist. Mit der Studie unterstützen sie die Bewertung der DpR als probates prognostisches Mittel zur frühzeitigen Erfassung von Patienten, die von einer Therapie profitieren. Allerdings handelt es sich um eine retrospektive, monozentrische Analyse mit sehr geringer Fallzahl, sodass Selektionseffekte nicht auszuschließen sind. Eine wesentliche Limitation liegt auch darin, dass ETS als prädiktiver Marker in dieser Analyse nicht erfasst wurde und die Kriterien dafür ( $\geq 20 \%$ Schrumpfung innerhalb von 8 Wochen nach Start der Therapie) in diesem Kollektiv nicht angewendet wurden.

\section{Fazit und Ausblick}

Trotz der Limitationen der Analyse unterstreicht diese Studie die Wertigkeit der DpR unter einer Erstlinientherapie als prädiktiver Marker für den Gesamtnutzen einer Therapie. Im Gegensatz zur etablierten alleinigen radiologischen Beurteilung des Ansprechens nach den RECIST-Kriterien konnten mehrere prospektive Studien zeigen, dass die tumordynamischen Parameter DpR und ETS die Sensitivität gegenüber einer Erstlinientherapie beim $\mathrm{mCRC}$ besser abbilden und prädiktiv für die 
Länge und Dauer von PFS und OS sind. Zukünftig könnten beide Parameter als neue, vielversprechende Endpunkte fungieren. Um beide Marker im klinischen Entscheidungsprozess zu etablieren, ist zunächst allerdings die prospektive Validierung von ETS und DpR in klinischen Studien notwendig.

\section{Referenzen}

1 Petrelli F, Pietrantonio F, Cremolini C, Di Bartolomeo M, Coinu A, Lonati V, de Braud F, Barni S: Early tumour shrinkage as a prognostic factor and surrogate end-point in colorectal cancer: a systematic review and pooled analysis. Eur J Cancer 2015;51:800-807.

2 Heinemann V, von Weikersthal LF, Decker T, Kiani A, Vehling-Kaiser U, AlBatran SE, Heintges T, Lerchenmüller C, Kahl C, Seipelt G, Kullmann F, Stauch M, Scheithauer W, Hielscher J, Scholz M, Müller S, Link H, Niederle $\mathrm{N}$, Rost A, Höffkes HG, Moehler M, Lindig RU, Modest DP, Rossius L, Kirchner T, Jung A, Stintzing S: FOLFIRI plus cetuximab versus FOLFIRI plus bevacizumab as first-line treatment for patients with metastatic colorectal cancer (FIRE-3): a randomised, open-label, phase 3 trial. Lancet Oncol 2014;15:1065-1075.
3 Heinemann V, Stintzing S, Modest DP, Giessen-Jung C, Michl M, Mansmann UR: Early tumour shrinkage (ETS) and depth of response (DpR) in the treatment of patients with metastatic colorectal cancer (mCRC). Eur J Cancer 2015;51:1927-1936.

4 Schwartzberg LS, Rivera F, Karthaus M, Fasola G, Canon JL, Hecht JR, Yu H, Oliner KS, Go WY: PEAK: a randomized, multicenter phase II study of panitumumab plus modified fluorouracil, leucovorin, and oxaliplatin (mFOLFOX6) or bevacizumab plus mFOLFOX6 in patients with previously untreated, unresectable, wild-type KRAS exon 2 metastatic colorectal cancer. J Clin Oncol 2014;32:2240-2247.

5 Cremolini C, Loupakis F, Antoniotti C, Lonardi S, Masi G, Salvatore L, Cortesi E, Tomasello G, Spadi R, Zaniboni A, Tonini G, Barone C, Vitello S, Longarini R, Bonetti A, D’Amico M, Di Donato S, Granetto C, Boni L, Falcone A: Early tumor shrinkage and depth of response predict long-term outcome in metastatic colorectal cancer patients treated with first-line chemotherapy plus bevacizumab: results from phase III TRIBE trial by the Gruppo Oncologico del Nord Ovest. Ann Oncol 2015;26:1188-1194.

Kontaktadresse: PD Dr. Sylvie Lorenzen, III. Medizinische Klinik, Klinikum rechts der Isar, Technische Universität München, Ismaninger Straße 22, 81675 München, Deutschland 